\title{
A Weak-Constraint-Based Data Assimilation Scheme for Estimating Surface Turbulent Fluxes
}

\author{
Jun Qin, Shunlin Liang, Senior Member, IEEE, Ronggao Liu, Hao Zhang, and Bo Hu
}

\begin{abstract}
Much attention has been focused on the assimilation of satellite data and products into land surface processes. In this letter, a variational data assimilation scheme is developed based on the weak-constraint concept. It assimilates surface skin temperature into a simple land surface model for the estimation of turbulent fluxes. An automatic differentiation technique is used to derive the adjoint codes to evaluate the gradient of the cost function. After the construction of this assimilation system, numerical experiments are conducted to test its performance with different model errors, and the comparison is also made with the strong constraint scheme. The results show that the land surface turbulent fluxes can be retrieved with highly satisfactory accuracy.
\end{abstract}

Index Terms-Data assimilation, evapotranspiration, land surface temperature (LST), weak constraint.

\section{INTRODUCTION}

A CCURATE estimation of energy and momentum fluxes, especially sensible and latent heat fluxes, between the land surface and the atmospheric boundary layer is required in a wide variety of agricultural, hydrological, and meteorological applications [1], [2]. Many methods, such as eddy correlation and Bowen ratio, can be used to measure these fluxes at the field scale. Their applicability, however, is limited on the fine spatial scale. At present, the only way to achieve this goal of mapping fluxes on a regional scale is to use remote sensing techniques that can provide various spatial and temporal imageries that cover large areas.

Estimation of surface energy balance components using remote sensing data can be roughly divided into three categories. Empirical methods directly build on the relationship between remote sensing products, such as various vegetation indexes and retrieved land surface temperature (LST) for the estimation. Residual methods of the energy budget couple some empirical formulas and physical mechanisms to realize the estimation of evapotranspiration ( $E T$ or $L E$ ) and sensible heat flux $(H)$

Manuscript received January 13, 2007; revised April 5, 2007. This work was supported in part by the Chinese Natural Science Foundation under Contract 40471098 and Contract 40601068, by the National 973 Program under Contract 2002CB4125, by the International Partnership Project under Contract CXTDZ2005-1, and by the National Aeronautics and Space Administration under Grant NNG05GD10G and Grant NAG512892.

J. Qin and R. Liu are with Institute of Geographic Science and Natural Resource, Chinese Academy of Sciences, Beijing 100101, China (e-mail: shuairenqin@gmail.com; liurg@ lreis.ac.cn).

S. Liang is with the Department of Geography, University of Maryland, College Park, MD 20742 USA (e-mail: sliang@geog.umd.edu).

H. Zhang is with the State Key Laboratory of Remote Sensing Science, Institute of Remote Sensing Application, Chinese Academy of Sciences, Beijing 100101, China (e-mail: zhangh.irsa@gmail.com).

B. Hu is with China Meteorological Administration, Beijing 100081, China.

Color versions of one or more of the figures in this paper are available online at http://ieeexplore.ieee.org.

Digital Object Identifier 10.1109/LGRS.2007.904004 by using remote sensing to directly estimate input parameters, such as the surface energy balance system [2]. In recent years, data assimilation methods have merged remote sensing data and soil-vegetation-atmosphere transfer models for estimating surface fluxes and have achieved encouraging results. This new method has received considerable attention from researchers in recent years [3]-[7], since it combines dynamic models and temporal remote sensing data based on the control theory to accurately retrieve critical parameters for flux estimation. The first two methods more or less use only the instantaneous data and empirical relationships.

A data assimilation system generally comprises the following three components [8]: 1) underlying dynamic model; 2) merging scheme; and 3) observation. Dynamic models are usually different in different disciplines, such as atmosphere, oceanography, and land surfaces. There exist many merging schemes. Some of them [9] are able to handle uncertainties both in models and observations, but others can consider the uncertainty only in observations. As a matter of fact, models are imperfect and always include uncertainties. It is important to consider this kind of uncertainty in the data assimilation scheme to improve the accuracy of estimation and prediction by a model. Kalman filters (KFs) and weak-constraint variational methods have attracted more and more attention from researchers since they can simultaneously handle uncertainties both in models and observations. Among the various kinds of KFs, the ensemble $\mathrm{KF}$ is the most popular since its application is relatively easy. However, it is more suitable for state estimation [10], [11]. If there are unspecified parameters in the model, especially timevarying parameters, which need to be estimated together with state variables, its application poses many limitations. Weakconstraint variational methods can estimate both state variables and model parameters. However, it is difficult to develop the adjoint model for the original one to evaluate the derivatives of the cost function with respect to control variables, which are composed of state variables and model parameters to be estimated. However, automatic differentiation (AD) techniques provide powerful tools to efficiently and effectively develop the adjoint of the model at the level of computer language codes.

In this letter, a variational land data assimilation scheme based on the weak-constraint concept [12]-[17] and AD techniques [18], [19] is proposed to assimilate LST to estimate surface turbulent fluxes by retrieving state variables and timevarying parameters at the same time. Numerical experiments are conducted to test the performance of this data assimilation system when different values of model uncertainties are used. Comparisons are also made to show the advantages of this weak-constraint variational scheme over the strong constraint variational scheme that assumes that the model is perfect. Estimation results indicate that this data assimilation 
scheme can effectively retrieve $L E$ and $H$ with a satisfactory accuracy.

This letter is organized as follows. In Section II, each component that comprises the data assimilation system is presented. Then, some numerical experiments are carried out to test the system. Finally, the conclusion is given.

\section{Construction of Data Assimilation System}

\section{A. Simple Land Surface Model}

The land surface model used in the study is similar to the one used by Caparrini et al. [5], [6]. However, it differs in two respects: one concerns the deep soil temperature, and the other is the parameterization of the sensible heat. The prognostic equations for LST $T_{s}$ and deep soil temperature $T_{d}$ are based on the classical force-restore method described as follows:

$$
\begin{aligned}
\frac{d T_{s}}{d t} & =\frac{2 \sqrt{\pi \omega}}{P}\left(R_{n}-H-L E\right)-2 \pi \omega\left(T_{s}-T_{d}\right) \\
T_{d} & =\sum_{i=1}^{D N} T_{s i} / D N
\end{aligned}
$$

where $R_{n}$ is the net radiation, $H$ is the sensible heat, $L E$ is the latent heat, $P$ is the thermal inertia of the land surface, $\omega$ is the diurnal frequency, $T_{s i}$ is the surface temperature for the $i$ th time step of the previous day, and $D N$ is the number of time steps in a day.

In (1), $H$ and $L E$ are parameterized as

$$
\begin{aligned}
H & =\rho c_{p} C_{H} U\left(T_{s}-T_{a}\right) \\
L E & =\frac{E F}{1-E F} H
\end{aligned}
$$

where $T_{a}$ is the air temperature, $\rho$ is the air density, $c_{p}$ is the air specific heat, $C_{H}$ is the bulk transfer coefficient for heat, $U$ is the wind speed, and $E F$ is the evaporative fraction. A different scheme that was adopted from the biosphere-atmosphere transfer scheme is used for the bulk transfer coefficient $C_{H}$, i.e.,

$$
\begin{aligned}
C_{H} & = \begin{cases}C_{H N}\left(1+24.5\left(-C_{H N} R i_{B}\right)^{1 / 2}\right), & R i_{B}<0 \\
C_{H N} /\left(1+11.5 R i_{B}\right), & R i_{B}>0\end{cases} \\
R i_{B} & =\frac{g}{\theta} \frac{\Delta \theta z_{\mathrm{ref}}}{\mathrm{U}^{2}} \\
C_{H N} & =\frac{\kappa^{2}}{\ln \left(z_{\mathrm{ref}} / z_{0 m}\right) \ln \left(z_{\mathrm{ref}} / z_{0 h}\right)}
\end{aligned}
$$

where $C_{H N}$ is the bulk heat transfer coefficient under neutral conditions, $R i_{B}$ is the bulk Richardson number, $g$ is the gravitational acceleration, $\theta$ is the potential temperature of the air, $\Delta \theta$ is the potential temperature difference between the air and the surface, $z_{\text {ref }}$ is the measurement height, and $z_{0 m}$ and $z_{0 h}$ are the roughness lengths for momentum and heat transfer, respectively.

From these formulas, it is noted that $C_{H N}$ and $E F$ are the two main factors [5], [6], [20] that directly affect the estimation of $H$ and $L E$. Thus, these two parameters will be retrieved as control variables in the process of data assimilation. Since the status of land surface slowly changes relative to meteorological forcing, $C_{H N}$ is assumed to be constant during one assimilation window. In other papers, it was assumed that EF is kept invariant during 09:00-18:00, local time, based on the analysis of some experimental data [5], [6], [21], [22]. In this study, the daily averaged EF is retrieved. Thus, the original assumption is relaxed. Values of $R_{n}, \rho, U$, and $T_{a}$ are taken from micrometeorological observations.

\section{B. Merging Scheme}

The merging scheme plays a key role in the entire data assimilation scheme since it determines how LST and the aforementioned land surface model are coupled. When using the variation method, two merging schemes that minimize a cost function exist. One is the strong constraint scheme, which treats the physical model as perfect, and the other is the weak constraint, which treats the physical model as imperfect. In this study, the weak-constraint method is formulated. In addition to those that were previously mentioned, control variables also include state variables of the model. Thus, a prognostic equation can be represented as after discretization in time, i.e.,

$$
\begin{aligned}
& T_{s}\left(t_{i}\right)=M\left(T_{s}\left(t_{i-1}\right)\right)+\varepsilon_{m}\left(t_{i}\right) \\
& T_{s}\left(t_{0}\right)=T_{s}^{b}+\varepsilon_{b} \\
& T_{o}\left(t_{k}\right)=T_{s}\left(t_{k}\right)+\varepsilon_{o}\left(t_{k}\right)
\end{aligned}
$$

where $M(\cdot)$ denotes the discrete dynamic model, $T_{s}^{b}$ is the background value for the initial surface temperature, $T_{o}$ is the observed LST, and $\varepsilon_{m}, \varepsilon_{b}$, and $\varepsilon_{o}$ are the model error with normal distribution, error for initial value of LST, and observation error, respectively, and they are assumed to be independent of each other. In this letter, $\varepsilon_{m}\left(t_{i}\right)$ and $\varepsilon_{o}\left(t_{k}\right)$ are assumed to be temporally independent.

The cost function $J$ can be represented as follows:

$$
\begin{aligned}
J= & \sum_{i=1}^{N}\left(T_{s}\left(t_{i}\right)-M\left(T_{s}\left(t_{i-1}\right)\right) \cdot Q_{m}^{-1}\right. \\
& \cdot\left(T_{s}\left(t_{i}\right)-M\left(T_{s}\left(t_{i-1}\right)\right)+\left(T_{s}\left(t_{0}\right)-T_{s}^{b}\right) \cdot Q_{b}^{-1}\right. \\
& \cdot\left(T_{s}\left(t_{0}\right)-T_{s}^{b}\right)+\sum_{k=1}^{K}\left(T_{o}\left(t_{k}\right)-T_{s}\left(t_{k}\right)\right) \cdot Q_{o}^{-1} \\
& \cdot\left(T_{o}\left(t_{k}\right)-T_{s}\left(t_{k}\right)\right)+\left(C_{H N}-C_{H N}^{b}\right) \cdot Q_{c}^{-1} \\
& \cdot\left(C_{H N}-C_{H N}^{b}\right)+\sum_{j=1}^{D}\left(E F_{j}-E F^{b}\right) \cdot Q_{e}^{-1} \\
& \cdot\left(E F_{j}-E F^{b}\right)
\end{aligned}
$$

where $N$ is the total number of time steps, $K$ is the number of observations, $D$ is the number of days in one assimilation window, $C_{H N}^{b}$ is the background value of $C_{H N}, E F^{b}$ is the background value of $E F, Q_{m}$ is the covariance of $\varepsilon_{m}, Q_{b}$ is the covariance of $\varepsilon_{b}, Q_{o}$ is the covariance for error of $T_{o}, Q_{c}$ is the covariance for error of $C_{H N}^{b}$, and $Q_{e}$ is the covariance for error of $E F^{b}$. The setting of these parameters in this letter will be described later.

The objective of the variational data assimilation method is to minimize the cost function $J$ to seek the most optimal control variables and, thus, accurately estimate $H$ and $L E$. There are two approaches to solve this optimization problem: one is the representer method [15], [17], and the other is the direct derivation of the derivatives of $J$ with respect to control variables and, thus, the use of the optimization algorithm [13]. The representer 


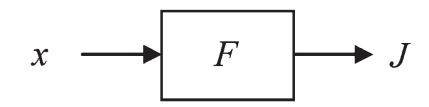

(a)

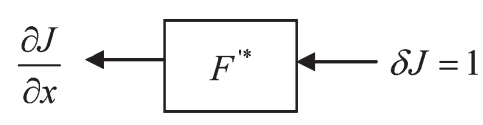

(b)

Fig. 1. Illustrations for the evaluation of derivatives of the cost function with AD techniques. $F$ denotes the whole codes for evaluating the cost function. $F^{\prime *}$ represents the adjoint codes of the original codes, which can be used to evaluate derivatives with ease.

method derives coupled Euler-Lagrange equations and then solves them. However, its formulation is very complicated. The second approach is easy to use. However, the process of manual derivation is tedious and time consuming. In this letter, $\mathrm{AD}$ techniques are used to develop adjoint codes for the evaluation of derivatives of the cost function $J$ at the level of computer source codes. AD is based on two facts [18], [23]. First, any computer code statement can be regarded as a composition of elementary functions. Second, the chain rule can be used to differentiate this composition of elementary functions. Many software packages have been developed in accordance with the principles described above for FORTRAN and C computer languages. In this study, the software package TAPENADE is applied to develop adjoint codes. The illustration of the AD running process is presented in Fig. 1. Provided that the derivatives of the cost function are evaluated, an optimization algorithm has to be chosen to minimize the cost function. In this letter, scaled conjugate gradient algorithms developed by Andrei [24] are used.

\section{LST}

In this letter, three different concepts of surface temperature occur: 1) radiometric surface temperature retrieved from remote sensing, which is used as the observation to be assimilated; 2) effective vegetated surface temperature, which is the state variable in the simple land surface model; and 3) aerodynamic surface temperature, which is used in the calculation of $H$ and $L E$. Assumptions have to be made in some studies for using these temperatures, having different physical meanings in a consistent way. Caparrini et al. [5] assume that the effective vegetated surface temperature modeled in the land surface model is the radiometric surface temperature, and thus, retrieved $C_{H N}$ is consistent with using radiometric LST in turbulent flux calculations. As a matter of fact, the difference between the physical meanings of these three temperatures can be considered as a part of model and observation errors in the framework of weak constraint. Therefore, no assumptions are needed about the physical meanings of quantities in the entire data assimilation system, and all variables and parameters have their original physical meanings. Observations $T_{o}$ are derived from the field infrared radiative thermometer in this study. The use of satellite-retrieved LST is the eventual goal.

\section{NuMERICAL EXPERIMENTS AND DISCUSSIONS}

After setting up the aforementioned data assimilation system, numerical experiments are performed to test its performance by
TABLE I

SETTINGS For The PARAMETERS IN THIS Data Assimilation System

\begin{tabular}{|c|c|c|c|}
\hline Symbol & Parameter & $\begin{array}{l}\text { Value or } \\
\text { average }\end{array}$ & Variance \\
\hline$P$ & $\begin{array}{l}\text { thermal inertia } \\
{\left[\mathrm{Jm}^{-2} \mathrm{~K}^{-1} \mathrm{~s}^{-1 / 2}\right]}\end{array}$ & 1000 & ----- \\
\hline$\omega$ & $\begin{array}{c}\text { diurnal frequency } \\
{\left[\mathrm{s}^{-1}\right]}\end{array}$ & $1 / 86400$ & ----- \\
\hline$C_{H N}^{b}$ & $\begin{array}{l}\text { background for } \\
\text { bulk transfer } \\
\text { coefficient for heat }\end{array}$ & 0.004 & $9.0 \mathrm{E}-6$ \\
\hline$E F^{b}$ & $\begin{array}{l}\text { background for } \\
\text { evaporative } \\
\text { fraction }\end{array}$ & 0.6 & 0.25 \\
\hline$\varepsilon_{m}$ & model error $\left[\mathrm{K}^{2}\right]$ & 0.0 & $1.0 \sim 9.0$ \\
\hline$\varepsilon_{o}$ & $\begin{array}{c}\text { observation error } \\
\qquad\left[\mathrm{K}^{2}\right]\end{array}$ & 0.0 & 2.0 \\
\hline$T_{s}^{b}$ & $\begin{array}{l}\text { background for } \\
\text { initial surface } \\
\text { temperature }[\mathrm{K}]\end{array}$ & 290 & 9.0 \\
\hline$T_{d}^{b}$ & $\begin{array}{l}\text { background for } \\
\text { initial deep } \\
\text { temperature }[\mathrm{K}]\end{array}$ & 290 & 9.0 \\
\hline
\end{tabular}
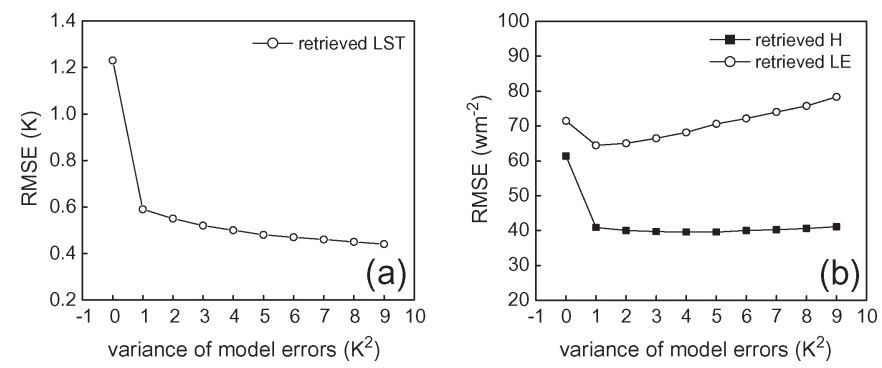

Fig. 2. Inversion results with increasing model errors from 0 to $9 \mathrm{~K}^{2}$. (a) RMSE of the retrieved LST. (b) RMSE of the retrieved $H$ and $L E$.

using field data, which are taken from the AmeriFlux Bondvill site. This site is located at $40.00^{\circ}$ latitude, $-88.29^{\circ}$ longitude, and its land cover is broadleaf crop. Measured micrometeorological variables and surface fluxes every $30 \mathrm{~min}$ from Julian day 231 to 260 in 1999 are used in this study, including air temperature, wind speed, air density, net radiation, temperature by infrared radiative thermometer, and sensible and latent heat fluxes. The length of one assimilation window is ten days, with five days overlap, and the total number of days in this assimilation experiment is 30 days. Parameters needed in this study, which are introduced in the second section, are set in Table I.

It is difficult to estimate the model errors, which are normally assigned according to experiences from researchers and a priori information. In this study, measurements of $1-9 \mathrm{~K}^{2}$ are taken with an increment of $1 \mathrm{~K}^{2}$ to investigate their effects on estimation results, as listed in Table I. In fact, the weak-constraint scheme will be close to the strong one when the model error takes a very minor value. Thus, the strong constraint scheme can be considered as a special case with a model error that is equal to $0 \mathrm{~K}^{2}$. Fig. 2 indicates the changes of the root mean square errors (RMSE) of LST, $H$, 

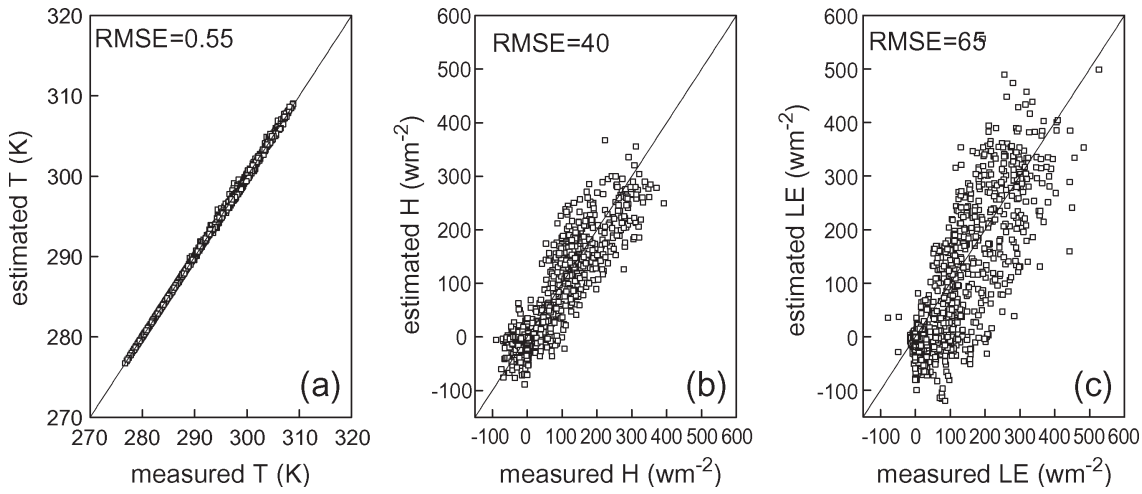

Fig. 3. Scatter plots for estimation results from data assimilation experiments. Measured versus estimated (a) surface temperature, (b) sensible heat flux, and (c) latent heat flux.
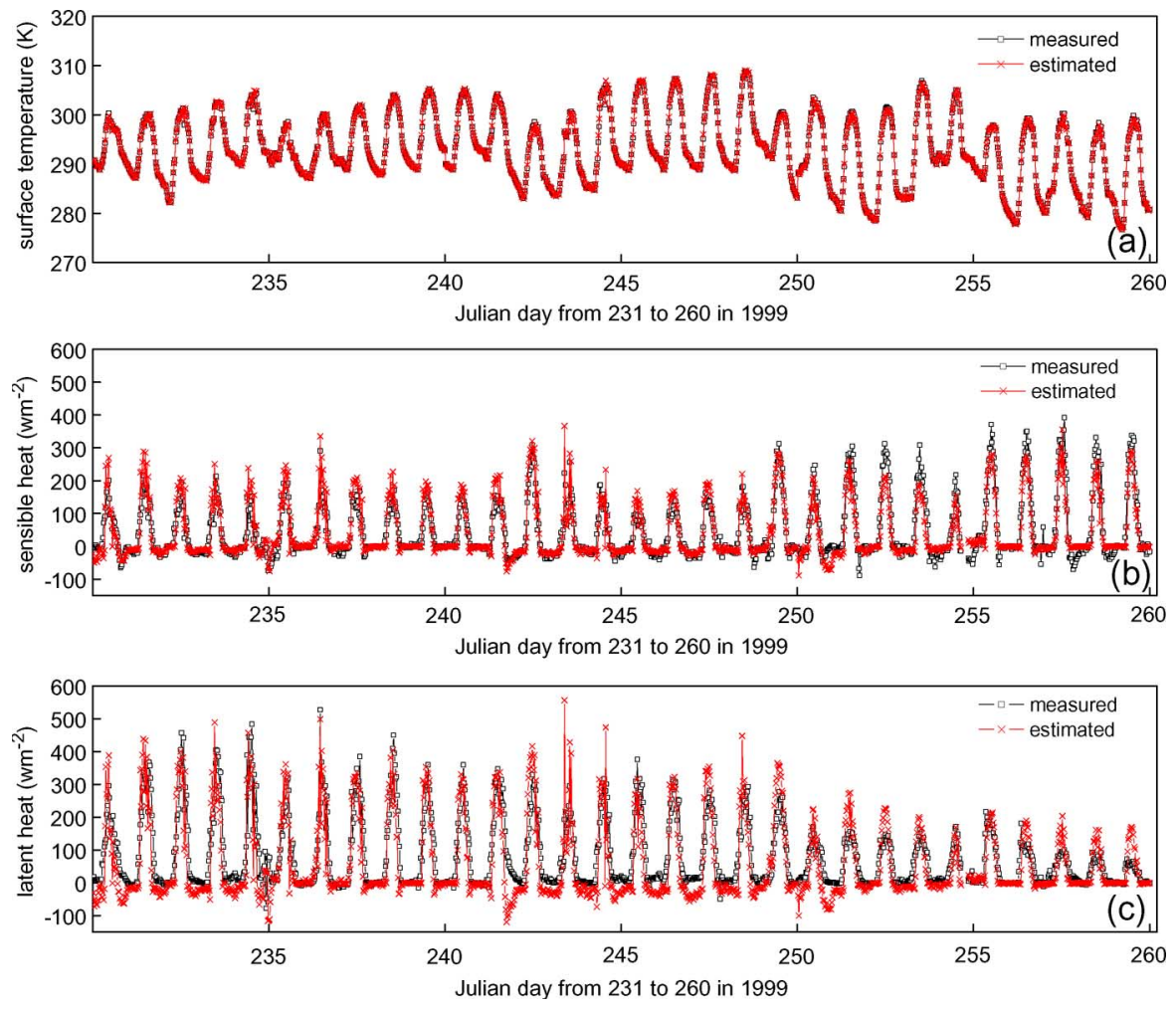

Fig. 4. Time profiles for estimation results and measurements. (a) Surface temperature. (b) Sensible heat flux. (c) Latent heat flux.

and $L E$, with increasing model errors from 0 to $9 \mathrm{~K}^{2}$. As seen, the weak-constraint scheme performs generally better than the strong one. The RMSE of the retrieved LST drops down with the variance of model errors ranging from 0 to $9 \mathrm{~K}^{2}$. The RMSE of the retrieved $H$ largely declines when the model error is considered and then becomes stable when the model error increases. The RMSE of the retrieved $L E$ first declines and then rises while the model error increases. This illustrates that the choice of the magnitude of the model error has an apparent effect on the retrieved latent heat flux. However, influences are not clear as far as other retrieved parameters are concerned.

Results, which correspond to the case where the model error is equal to $2 \mathrm{~K}^{2}$, are presented in Figs. 3(a) and 4(a). They show that measured and estimated surface temperatures match very well and that the RMSE is $0.55 \mathrm{~K}$. Figs. 3(b) and 4(b) indicate that the data assimilation method developed can estimate the sensible heat flux with satisfactory accuracy; the RMSE is about $40 \mathrm{~W} \cdot \mathrm{m}^{-2}$, considering the error in $L E$ and $H$ measured in the field to be around $50 \mathrm{~W} \cdot \mathrm{m}^{-2}$. Retrieved latent heat fluxes are not as good as sensible heat fluxes, as shown in Figs. 3(c) and 4(c), with an RMSE equal to about $65 \mathrm{~W} \cdot \mathrm{m}^{-2}$. In particular, estimated $L E$ values are systematically biased low largely during the nighttime of Julian days 242 and 251, and several $L E$ values during the daytime of Julian days 244 and 249 are biased high. Control variables, including modeling effective surface temperature, bulk transfer coefficient for heat, and daily averaged evaporative fraction, are adjusted to minimize mostly the misfit between modeling surface temperature and its observed counterpart. As a result, accurate surface temperatures are retrieved and so do sensible heat fluxes in accordance with (3). Since daily averaged EF are retrieved in this study and approximate constant EF are held only around the local noon [21], [22], large biases will occur when patterns greatly differ 

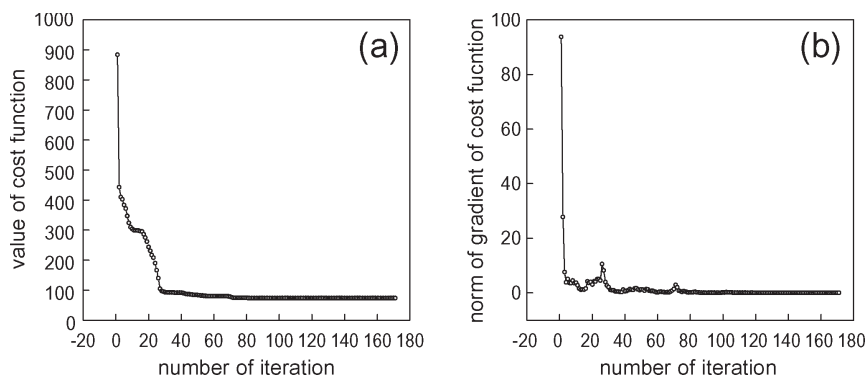

Fig. 5. Performance of cost function and its gradient in the course of minimization for a period from Julian day 231 to 240 . (a) Value of the cost function. (b) Norm of the gradient of the cost function.

between $L E$ and $H$. The discussions above indicate that the relatively high RMSE of the retrieved latent heat flux stems from some systematic errors since the parameterization for water balance processes that are closely related to evapotranspiration is rather simple in this letter. However, the whole retrieved results are encouraging, although the RMSE of the retrieved latent heat flux is a little high compared to the aforementioned measurement uncertainties since there is no additional information introduced, such as soil texture and land cover type, in addition to the observed LST and a few meteorological variables.

Fig. 5 shows the performance of minimization of the cost function for a period from Julian day 231 to 240 in the case above. It can be seen that the optimization works effectively and efficiently. Both the value of the cost function and the norm of its gradient rapidly decline with the increase in the number of iteration cycles and reach stability after roughly 80 iterations. Minimizations in other assimilation windows show similar patterns.

\section{CONCLUSION}

Quantitative estimation of turbulent fluxes between atmospheric boundary layer and land surface plays an important part in a wide range of applications. The data assimilation method has received more and more attention because of its advantages over other estimation methods. In this study, a simple land surface model has been developed based on the work by Caparrini et al. The variational data assimilation method was developed using the weak-constraint concept. Rather than using the representer method or manual derivation, direct evaluation of derivatives of the cost function through $\mathrm{AD}$ techniques was applied. This can greatly accelerate the speed of the data assimilation system. After the construction of the data assimilation system, different model errors were used to test it. The retrieval values were validated against the field measurements, and the results were very encouraging. The RMSEs for sensible and latent heat fluxes were around 40 and $60 \mathrm{~W} \cdot \mathrm{m}^{-2}$, respectively. The eventual goal was to use this algorithm with satellite data on a regional scale. Much work needs to be done to improve this data assimilation system, such as the comparison between different optimization algorithms and the replacement of the land surface model used in this study with a more advanced and complex one. Research along these lines is on the way.

\section{ACKNOWLEDGMENT}

The authors would like to thank Prof. Y. Dai for his help with land surface modeling and three anonymous reviewers for their comments and suggestions on the earlier versions of this letter.

\section{REFERENCES}

[1] D. Courault, B. Seguin, and A. Olioso, "Review on estimation of evapotranspiration from remote sensing data: From empirical to numerical modeling approaches," Irrig. Drain. Syst., vol. 19, no. 3/4, pp. 223-249, Nov. 2005.

[2] Z. Su, "The surface energy balance system (SEBS) for estimation of turbulent heat fluxes," Hydrol. Earth Syst. Sci., vol. 6, no. 1, pp. 85100, 2002.

[3] G. Boni, F. Castelli, and D. Entekhabi, "Sampling strategies and assimilation of ground temperature for the estimation of surface energy balance components," IEEE Trans. Geosci. Remote Sens., vol. 39, no. 1, pp. 165172, Jan. 2001

[4] G. Boni, F. Castelli, and D. Entekhabi, "Land data assimilation with satellite measurements for the estimation of surface energy balance components and surface control on evaporation," Water Resour. Res., vol. 37, no. 6, pp. 1713-1722, 2001.

[5] F. Caparrini and F. Castelli, "Estimation of surface turbulent fluxes through assimilation of radiometric surface temperature sequences," J. Hydrometeorol., vol. 5, no. 1, pp. 145-159, Feb. 2004.

[6] F. Caparrini, F. Castelli, and D. Entekhabi, "Mapping of land-atmosphere heat fluxes and surface parameters with remote sensing data," Bound. Layer Meteorol., vol. 107, no. 3, pp. 605-633, Jun. 2003.

[7] F. Castelli, D. Entekhabi, and E. Caporali, "Estimation of surface heat flux and an index of soil moisture using ajoint-state surface energy balance," Water Resour. Res., vol. 35, pp. 3115-3125, 1999.

[8] P. F. J. Lermusiaux and A. R. Robinson, "Data assimilation in models," in Encyclopedia of Ocean Sciences. New York: Academic, 2001, pp. 623-634.

[9] J. M. Lewis, S. Lakshmivarahan, and S. K. Dhall, Dynamic Data Assimilation: A Least Squares Approach. Cambridge, U. K.: Cambridge Univ. Press, 2006.

[10] J. D. Annan and J. C. Hargreaves, "Efficient parameter estimation for a highly chaotic system," Tellus, vol. 56A, no. 5, pp. 520-526, Oct. 2004.

[11] G. A. Kivman, "Sequential parameter estimation for stochastic systems," Nonlinear Process. Geophys., vol. 10, no. 3, pp. 253-259, 2003.

[12] G. A. Kivman, "Weak constraint data assimilation for tides in the Arctic Ocean," Prog. Oceanogr., vol. 40, no. 1, pp. 179-196, 1997.

[13] L.-J. Natvik, M. Eknes, and G. Evensen, "A weak constraint inverse for a zero-dimensional marine ecosystem model," J. Mar. Syst., vol. 28, no. 1, pp. 19-44, Feb. 2001.

[14] G. Evensen and N. Fario, "Solving for the generalized inverse of the Lorenz model," J. Meteorol. Soc. Jpn., vol. 75, no. 1B, pp. 229-243, 1997.

[15] B. S. Chua and A. F. Bennett, "An inverse ocean modeling system," Ocean Model., vol. 3, no. 3, pp. 137-165, 2001.

[16] F. Uboldi and M. Kamachi, "Time-space weak-constraint data assimilation for nonlinear models," Tellus, vol. 52A, no. 4, pp. 412-421, Aug. 2000.

[17] L. Xu and R. Daley, "Towards a true 4-dimensional data assimilation algorithm: Application of a cycling representer algorithm to a simple transport problem," Tellus, vol. 52A, no. 2, pp. 109-128, Mar. 2000.

[18] R. Giering and T. Kamimski, "Recipes for adjoint code construction," ACM Trans. Math. Softw., vol. 24, no. 4, pp. 437-474, Dec. 1998.

[19] A. Verma, "An introduction to automatic differentiation," Curr. Sci., vol. 78, no. 7, pp. 804-807, 2000.

[20] W. T. Crow and W. P. Kustas, "Utility of assimilation surface radiometric temperature observations for evaporative fraction and heat transfer coefficient retrieval," Bound. Layer Meteorol., vol. 115, pp. 105-130, 2005.

[21] R. D. Crago, "Conservation and variability of the evaporative fraction during the daytime," J. Hydrol., vol. 180, no. 1, pp. 173-194, May 1996.

[22] R. D. Crago and W. Brutsaert, "Daytime evaporation and self-preservation of the evaporative fraction and the Bowen ration," J. Hydrol., vol. 178, no. 1, pp. 241-255, Apr. 1996.

[23] C. Faure, "Adjoint strategies for multi-layered programs," Optim. Methods Softw., vol. 17, no. 1, pp. 129-164, Jan. 2002.

[24] N. Andrei, SCALCG: A Nonlinear Scaled Conjugate Gradient Algorithm for Unconstrained Optimization. Bucharest, Romania: Res. Inst. Informat., Center Adv. Model. Optim., 2005. 